\title{
Simulation of Bulk Evaporation and Condensation using the Energy of Fluid Method
}

\author{
Elijah Gasmen ${ }^{1}$ \\ University of Memphis, Memphis, Tennessee, 38111, United States
}

\begin{abstract}
Cryogenic propellant tanks absorb solar radiation during long duration space missions, which heats the liquid propellant. The tank self-pressurizes due to the evaporation of the liquid propellant. The design of these cryogenic storage systems relies on the prediction of selfpressurization within the container. The overall objective of this research is to simulate evaporation and condensation within a self-pressurizing tank by implementing the Energy of Fluid (EOF) method in commercial computational fluid dynamics software. The EOF method is used to model conjugate heat transfer and phase change. Results of the simulations are compared to the pressure, temperature, and phase histories obtained from the experiment.
\end{abstract}

\section{Nomenclature}

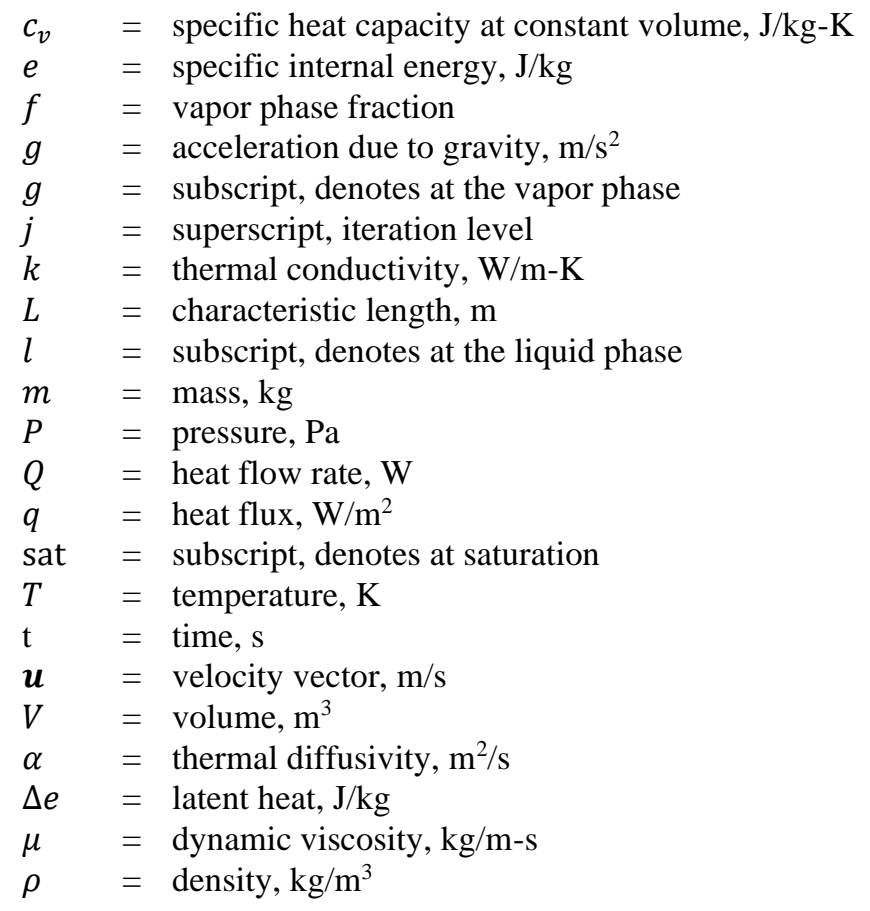

\section{Introduction}

The liquid contents of cryogenic propellant tanks experience evaporation due to heating from incident solar radiation [1]. The accumulation of vapor causes a pressure rise within the tank. This process is called self-

\footnotetext{
${ }^{1}$ Graduate Research Assistant, Department of Mechanical Engineering, AIAA Student Member.
} 
pressurization and may cause the tank to rupture if left uncontrolled [2]. The design of a tank is dependent on the prediction of this process and various methods to control it. This research focuses on the prediction of selfpressurization.

\section{A. Background}

The case investigated is the closed fuel tank experiment conducted in the S-IVB stage of vehicle AS203 in 1966 [2]. The objective of the experiment is to determine the pressure rise of the closed liquid hydrogen fuel tank in orbit. At the beginning of the experiment, all valves connected to the LH2 tank are closed, and the vehicle continues to accelerate to keep the liquid settled at the aft end. Approximately $7257 \mathrm{~kg}$ of LH2 is present in the tank at a pressure of $85,495 \mathrm{~Pa}$. The tank self-pressurizes as heat from solar radiation causes the hydrogen to evaporate. The pressure at the end of the experiment is $259,932 \mathrm{~Pa}$. The duration of the experiment is $5360 \mathrm{sec}$, which corresponds to a pressure rise rate of approximately $32.5 \mathrm{~Pa} / \mathrm{s}$.

This experiment is the subject of several simulation attempts to predict the pressure rise. A summary of results from the following cases is shown on Fig. 1. Ref. [2] employs a simplified thermodynamic model of the tank, which separates the fluid domain into a homogenous ullage section and a homogenous liquid section with separate heating rates. This model predicted a lower pressure rise than the experiment at $27.7 \mathrm{~Pa} / \mathrm{s}$. The study concludes that accurate prediction of the pressure rise requires both ullage and liquid heating rates as well as modeling of thermal stratification within the tank.

Ref. [3] calculates the heat flow into the tank using absorptivity of the tank walls. Two programs are used to predict the pressure rise within the tank using these heat flow rates. The first program simulates a single node ullage and a single node liquid. This program reports no evaporation in the liquid and lacks stratification in the model. The program predicts a higher pressure near the beginning of the experiment and a lower pressure near the end. The second program includes evaporation in the liquid. The result slightly overpredicts the pressure at 262,690 $\mathrm{Pa}$ and predicts a boil-off of $14.51 \mathrm{~kg}$. While these programs predicted the pressure rate within the desired tolerance, thermal stratification is unaccounted for in the models.

A more recent study by Ref. [1] uses commercial computational fluid dynamics (CFD) software enhanced by the energy of fluid approach to model the pressure rise in the AS203 fuel tank. The energy equation implemented into the software features transient conduction phase change but lacks convection. The model predicts an end pressure of 239,459 $\mathrm{Pa}$ and condensation within the ullage, unlike the previous models mentioned. Temperature and phase contours show that superheated vapor, saturated fluid, and compressed liquid all occur in the tank.

\section{B. Objective}

The objective of this research is to reproduce the results obtained by Ref. [1] and improve upon the model by adding convection heat transfer. Thermal stratification and destratification can be modeled with the addition of natural convection, which will result in more accurate results. Computational time is a limiting factor on the progress of simulations. Ref. [1] discretizes the energy equation within the code itself, uses a third-party matrix solver to find the temperature solution, and can only utilize serial processing. The current research will utilize the built-in scalar transport equation solver in the software and utilize parallel processing nodes, which will substantially decrease the computational time. The current research will also show no change in results for the verification cases. Simulation of the AS203 tank experiment will be reconducted using this implementation before adding convection.

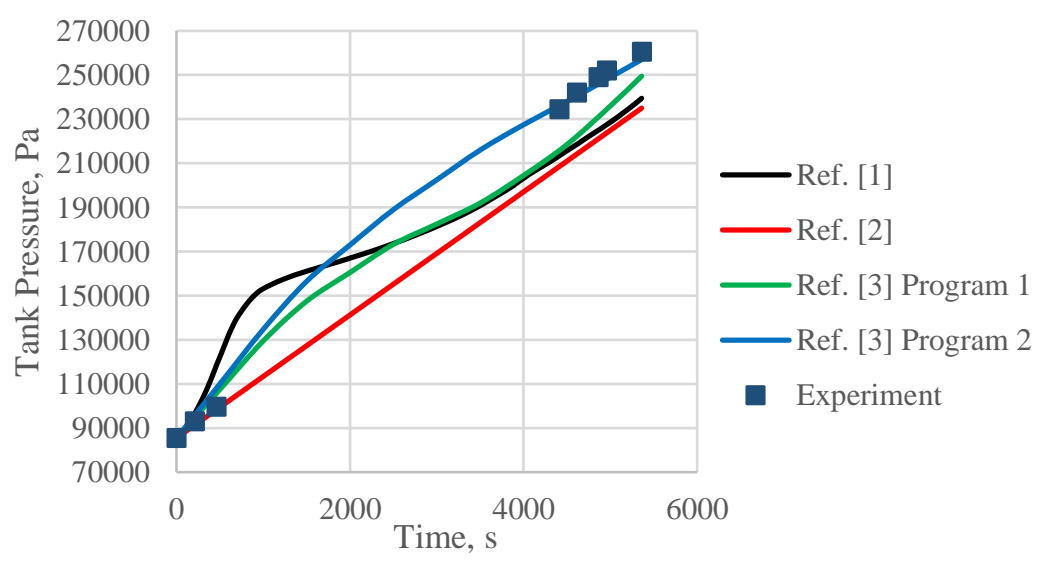

Fig. 1 Summary of previous pressure rise predictions for AS203 closed fuel tank experiment. 


\section{Computational Setup}

Commercial CFD software is used to simulate the fluid flow and heat transfer [10]. This software uses the finite volume method to solve the unsteady continuity and momentum equations, along with custom user defined scalar (UDS) transport equations [11-13]. The software utilizes the algebraic multigrid (AMG) method to solve the discretized equations. For the current research, all simulations use the semi-implicit-method-for-pressure-linkedequations (SIMPLE). For spatial discretization, gradients are evaluated using the least-squares-cell-based method, pressure is evaluated with the Standard method, momentum is evaluated with a Second Order Upwind method, and energy is evaluated with a First Order Upwind method. First order implicit transient formulation is used to progress through time.

\section{A. Governing Equation}

The energy of fluid method uses an internal energy formulation to solve the energy equation. Developed by the investigators of Ref. [5], this approach is similar in concept to the enthalpy formulation already present in the software [12]. Research shows that the two methods produce the same results in similar computational times $[5,6]$. However, the energy of fluid method reduces complexity by omitting the transient pressure term present in the enthalpy formulation. The governing equation given by the energy of fluid approach is Eq. (1) [5].

$$
\rho \frac{\partial e}{\partial t}+\nabla \cdot(\rho \boldsymbol{u} e)=\nabla \cdot(k \nabla T)
$$

In addition, Eq. (2) calculates the piecewise linear specific internal energy of a cell using the latent heat and sensible heat, assuming constant properties [4-6].

$$
e=c_{v, l}\left(T+f\left(T_{\text {sat }}-T\right)\right)+f \Delta e+c_{v, g} f\left(T-T_{\text {sat }}\right)
$$

The vapor phase fraction of a cell is zero for a liquid and one for a vapor. The phase fraction of a mixed phase cell is calculated using Eq. (3).

$$
f=\frac{e-e_{l}}{e_{g}-e_{l}}
$$

From Eqs. (1) and (2), a form of the energy equation that includes the latent heat is given in Eq. (4) [4]. When convection is not present, the equation is reduced to Eq. (5).

$$
\begin{aligned}
\rho c_{v, i} \frac{\partial T}{\partial t}+\nabla \cdot\left(\rho \boldsymbol{u} c_{v, i} T\right) & =\nabla \cdot(k \nabla \mathrm{T})-\rho \Delta e \frac{\partial f}{\partial t}-\Delta e \nabla \cdot(\rho \boldsymbol{u} f) \\
\rho c_{v, i} \frac{\partial T}{\partial t} & =\nabla \cdot(k \nabla \mathrm{T})-\rho \Delta e \frac{\partial f}{\partial t}
\end{aligned}
$$

This approach allows both phases to be solved using a single governing equation and results in a more gradual phase transition at the interface. Updating the phase fraction using the internal energy values and latent heat of vaporization is called the E-based update method. This update method is different to the T-based update method, which uses a predetermined temperature interval centered around the saturation temperature to model the phase change interface [5,6]. Research shows that the E-based method is superior to the T-based method in terms of computational time, stability, and accuracy $[5,6]$.

\section{B. Code Implementation}

To use the energy of fluid method in the simulations, a user defined function (UDF) is implemented into the software [13]. The UDF contains a library of functions and variables required to model each case. The UDS transport equation solver in the software requires these custom functions to specify values in the governing equation. Each case requires a customized UDF because of the different materials and circumstances that pertains to that specific case.

The iteration loop for the flow variables consists of the following steps. The total system mass is calculated at initialization and remains constant as described by Eq. (6). Mass, velocity, and temperature solutions are obtained by the software. Afterward, the mass of the liquid phase is calculated. The decrease in the liquid mass due to evaporation corresponds to the increase in vapor mass. The current vapor mass is the difference between the total system mass at initialization and the current liquid mass. The bulk vapor density and temperature are calculated using a volume weighted average, given in Eqs. (7) and (8). These values are used to calculate saturation pressure and temperature of 
the fluid. The cell internal energy is calculated using the saturation pressure and cell temperature. The cell phase fraction is updated using the E-based method, and material properties are updated using all the newly calculated values. The iteration loop repeats until convergence is achieved.

$$
\begin{gathered}
m_{\text {total }}=m_{g}+m_{l}=\text { constant } \\
\rho_{\text {bulk }}=\frac{\sum\left(m_{g}\right)_{\text {cell }}}{\sum(V f)_{\text {cell }}} \\
T_{\text {bulk }}=\frac{\sum(T V f)_{\text {cell }}}{\sum(V f)_{\text {cell }}}
\end{gathered}
$$

This update sequence as implemented in the software is detailed in Fig. 2. The software slightly reorders the sequence [13]. Despite this modification, the reordering is ultimately negligible when solution residuals progress towards zero.

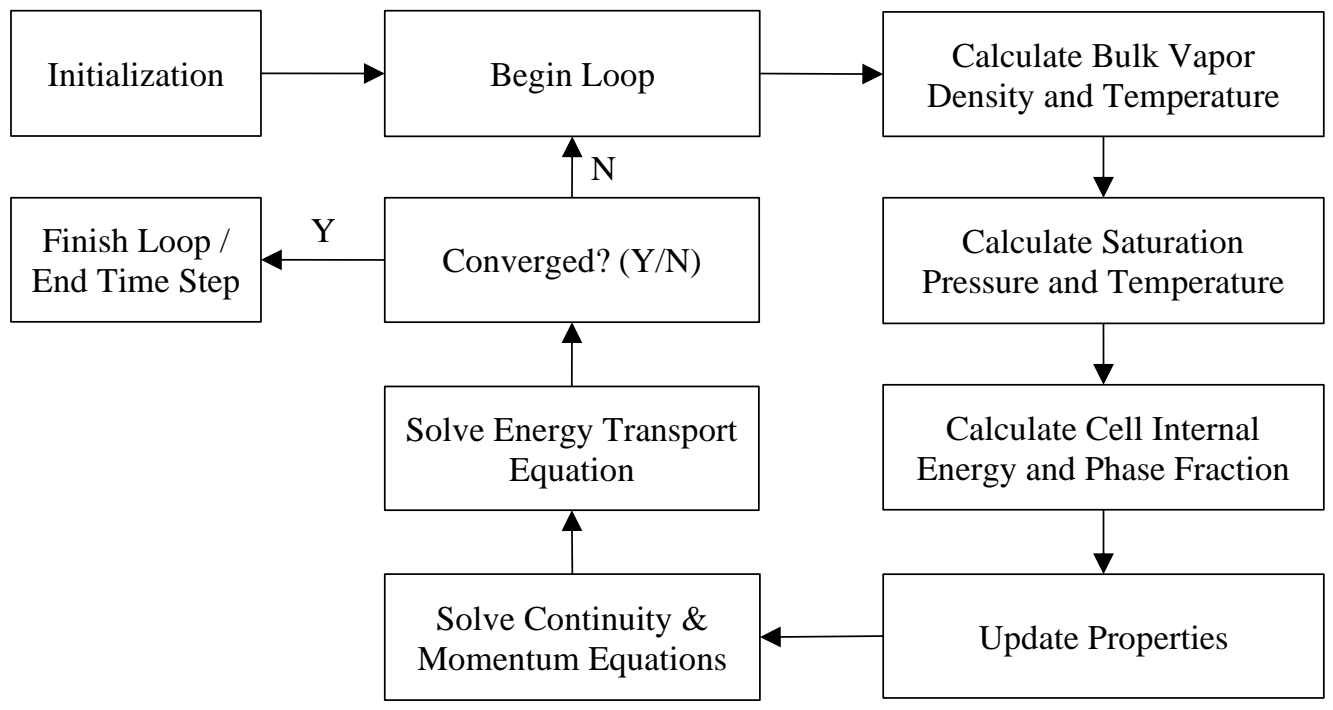

Fig. 2 Solver iteration loop.

The residuals for the saturation pressure, evaporated liquid fraction (ELF), and phase fraction are calculated using error formulas given by Eqs. (9) and (10). The ELF is discussed in Section V. Originally, the maximum change in phase fraction of a cell is used to judge convergence. However, this form often leads to an oscillating convergence. A more tolerant form to judge phase fraction convergence uses the change in the sum of mixed phase fraction values. Additionally, solution convergence is judged using solver residuals provided by the software for the continuity, momentum, and energy equations. The tolerances enforced for convergence are $1 \mathrm{E}-3$ for continuity and momentum, $1 \mathrm{E}-9$ for energy, and 1E-6 for the saturation pressure, ELF, and phase fraction.

$$
\begin{gathered}
\operatorname{residual}\left(P_{\text {sat }}\right)=\frac{\left|P_{\text {sat }}^{j}-P_{\text {sat }}^{j-1}\right|}{P_{\text {sat }}^{j-1}} \\
\text { residual }(\mathrm{ELF})=\left|\frac{\mathrm{ELF}^{j}-\mathrm{ELF}^{j-1}}{\mathrm{ELFF}^{j-1}}\right| \\
\text { residual }(f)=\frac{\left|\sum\left(f_{0<f<1}\right)^{j}-\sum\left(f_{0<f<1}\right)^{j-1}\right|}{\sum\left(f_{0<f<1}\right)^{j-1}}
\end{gathered}
$$




\section{Code Verification}

Code verification conducted in Ref. [1] consists of three cases. These cases confirm that the method is accurate with published analytical results. Reproduction of these results is meant to show that the new implementation of the UDF provides the same results. Water is used as the heat transfer medium in all three cases. The first two cases investigate unsteady conduction heat transfer. The third case investigates unsteady conduction phase change.

\section{A. Unsteady Conduction Heat Transfer}

The governing differential equation used to model one-dimensional conduction heat transfer is shown in Eq. (12). The following two cases are used to verify the implementation this equation.

$$
\rho c_{v} \frac{\partial T}{\partial t}=k \frac{\partial^{2} T}{\partial y^{2}}
$$

For both cases, the same $0.1 \mathrm{~m} \times 0.1 \mathrm{~m}$ two-dimensional square geometry is used in a uniform $100 \times 100$ cell computational mesh. The simulations are run to a flow time of $600 \mathrm{sec}$ at a time step size of $1 \mathrm{sec}$. Each time step is iterated until the solution residuals are converged according to the previously stated criteria in Section III Part B. The only solution residual monitored in these cases is the energy equation. Table 1 details the constant material properties of water used in the simulations, which are at a temperature of $300 \mathrm{~K}$. The saturation temperature of water is 373.15 $\mathrm{K}$ at a pressure of 101,325 $\mathrm{Pa}$. Therefore, no phase change occurs.

Table 1 Material properties of liquid water at 101,325 Pa and $300 \mathrm{~K}$.

\begin{tabular}{|c|c|c|c|}
\hline Thermal Conductivity & Density & Specific Heat & Thermal Diffusivity \\
\hline $\boldsymbol{k}, \mathbf{W} / \mathbf{m}-\mathbf{K}$ & $\boldsymbol{\rho}, \mathbf{~ k g} / \mathbf{m}^{\mathbf{3}}$ & $\boldsymbol{C}_{\boldsymbol{v}}, \mathbf{J} / \mathbf{k g}-\mathbf{K}$ & $\boldsymbol{\alpha}, \mathbf{m}^{2} / \mathbf{s}$ \\
\hline 0.6102 & 996.5298 & 4130.0 & $1.4646 \mathrm{E}-7$ \\
\hline
\end{tabular}

\section{Temperature Boundary Condition}

An analytical solution for the temperature at a distance $y$ at time $t$ for a region of $0<y<L$ is provided by Eq. (13) [7].

$$
T(y, t)=T_{0}-\left(T_{0}-T_{L}\right) \frac{y}{L}-\frac{2}{\pi}\left(T_{0}-T_{L}\right) \sum_{z=1}^{\infty}\left[\frac{1}{z} \sin \left(z \pi \frac{y}{L}\right) \exp \left(-z^{2} \pi^{2} \frac{\alpha t}{L^{2}}\right)\right]
$$

The boundary conditions are detailed in Fig. 2a. Along the top wall, the temperature boundary condition is $300 \mathrm{~K}$. At the bottom wall, the temperature boundary condition is $325 \mathrm{~K}$. The two side walls are adiabatic. The initial temperature of the fluid is $300 \mathrm{~K}$. Fig. $2 \mathrm{~b}$ shows the results of the simulation at $600 \mathrm{sec}$, which are in good agreement with the analytical solution.

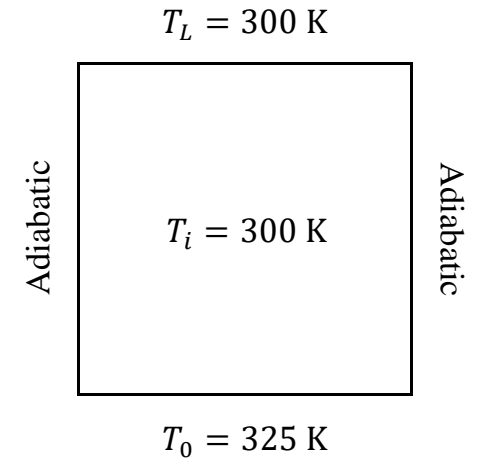

(a)

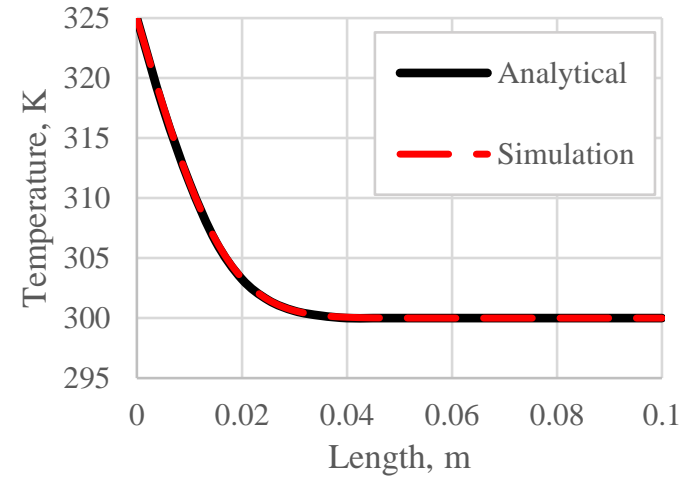

(b)

Fig. 3 Temperature boundary condition case: wall boundary conditions (a) and temperature distribution at $600 \mathrm{sec}(\mathrm{b})$. 


\section{Heat Flux Boundary Condition}

An analytical solution for this situation is provided by Eq. (14) [8]. The time dependent temperature is calculated as

$$
T(y, t)=T_{i}+\frac{2 q(\alpha t)^{1 / 2}}{k} \sum_{z=0}^{\infty}\left[\operatorname{ierfc} \frac{(2 z+1)(L-y)}{2(\alpha t)^{1 / 2}}+\operatorname{ierfc} \frac{(2 z+1)(L+y)}{2(\alpha t)^{1 / 2}}\right]
$$

where

$$
\operatorname{ierfc}(x)=\frac{1}{\sqrt{\pi}} e^{-x^{2}}-x[\operatorname{ierfc}(x)]
$$

The boundary conditions are detailed in Fig. 3a. At the top wall, the heat flux boundary condition is $200 \mathrm{~W} / \mathrm{m}^{2}$. All other walls are adiabatic. The fluid is initialized at $300 \mathrm{~K}$. Fig. $3 \mathrm{~b}$ shows the results from the simulation at 600 sec, which are in good agreement with the analytical solution.

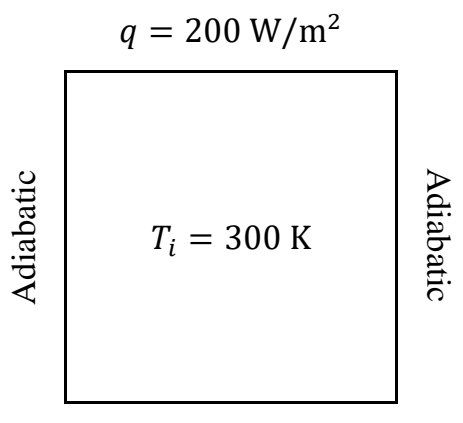

Adiabatic

(a)

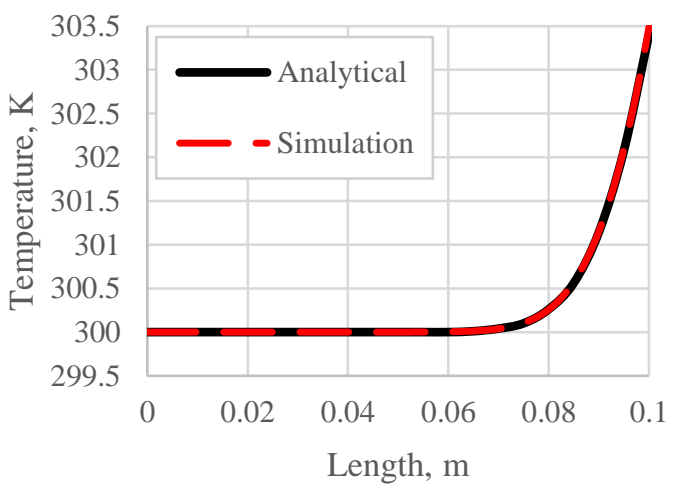

(b)

Fig. 4 Heat flux boundary condition case: wall boundary conditions (a) and temperature distribution at $600 \mathrm{sec}(\mathrm{b})$.

\section{B. Conduction Phase Change}

The case described in this part of the section is used to verify the phase change implementation of the model. Phase change in this case is one of solidification and melting and occurs purely due to conduction heat transfer. An analytical solution for this one-dimensional solidification case is given as

$$
Y=2 \lambda(\alpha t)^{1 / 2}
$$

where $\lambda=0.79$ and $\alpha$ is of the solid phase [8]. Table 2 shows the relevant material properties of the fluid. The freezing point is set at $275.15 \mathrm{~K}$ with a latent heat value of $334,944 \mathrm{~J} / \mathrm{kg}$.

Table 2 Material properties of liquid and solid water at 101,325 Pa.

\begin{tabular}{|c|c|c|c|c|}
\hline \multirow{2}{*}{ Phase } & Thermal Conductivity & Density & Specific Heat & Thermal Diffusivity \\
\cline { 2 - 5 } & $\boldsymbol{k}, \mathbf{W} / \mathbf{m}-\mathbf{K}$ & $\boldsymbol{\rho}, \mathbf{~ k g} / \mathbf{m}^{\mathbf{3}}$ & $\boldsymbol{C}_{\boldsymbol{v}}, \mathbf{J} / \mathbf{k g}-\mathbf{K}$ & $\boldsymbol{\alpha}, \mathbf{~ m}^{2} / \mathbf{s}$ \\
\hline Liquid & 0.6029 & 1000.0 & 4186.8 & $1.4400 \mathrm{E}-7$ \\
\hline Solid & 2.2190 & 920.0 & 2101.8 & $1.1476 \mathrm{E}-6$ \\
\hline
\end{tabular}

The boundary conditions are detailed in Fig. 5a. The top wall temperature is $275.15 \mathrm{~K}$. The bottom wall temperature is $273.15 \mathrm{~K}$, which is where solidification originates. The side walls are adiabatic. The initial fluid temperature is $275.15 \mathrm{~K}$. The simulations are run to a flow time of $600 \mathrm{sec}$ using a time step size of $1 \mathrm{sec}$. The solution residuals monitored in this case are the energy equation and the mixed phase fraction as shown in Eq. (11).

A mesh convergence study is conducted using a $0.0025 \mathrm{~m} \mathrm{x} 0.1 \mathrm{~m}$ two-dimensional geometry of varying mesh sizes. The results of study are shown on Fig. $5 \mathrm{~b}$ alongside the results from the analytical solution. The legend denotes 
grid size. As the mesh is refined, the numerical solution nearly converges onto the analytical solution. The results are shown to be in good agreement and are nearly exact to the results obtained in Ref. [1].

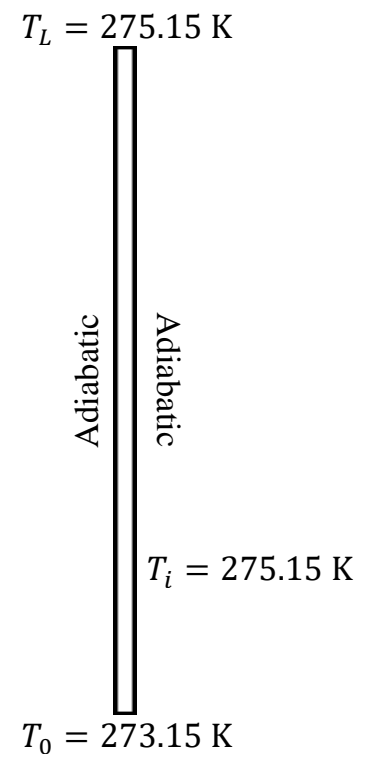

(a)

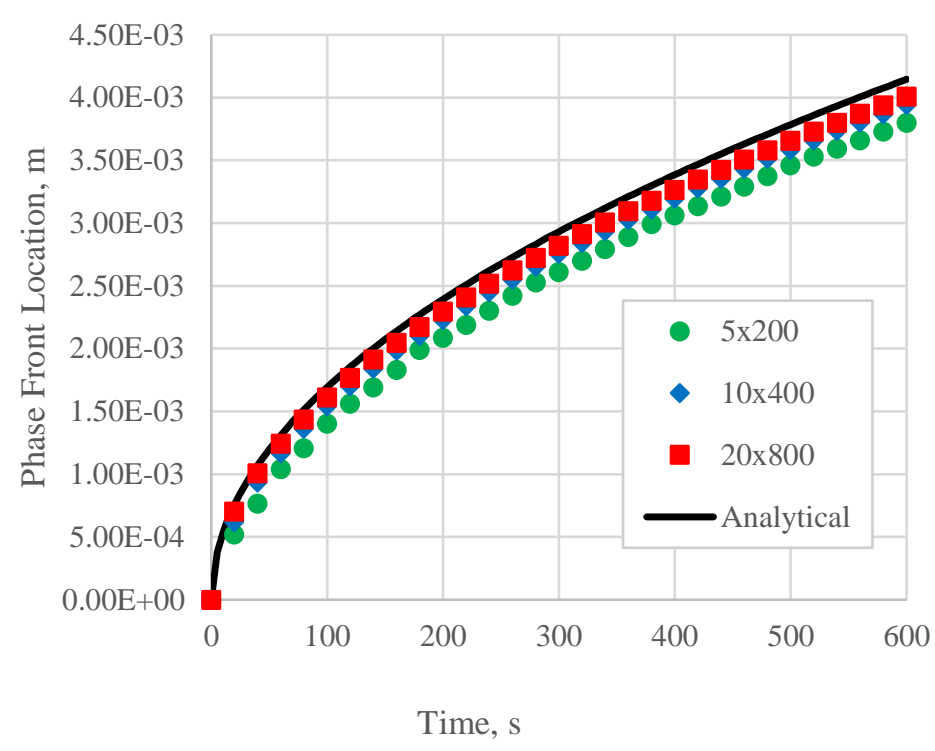

(b)

Fig. 5 Conduction phase change case: boundary conditions (a) and phase front location (b).

\section{Code Validation}

In the verification cases, material properties are constant with temperature and only change with phase. The saturation pressure and temperature also do not change. The specific internal energy is calculated with this assumption. Simulation of a vapor is substantially more complex because the properties are dependent on temperature and pressure. As a result, Eq. (2) is insufficient in calculating the specific internal energy at either phase. Instead, material properties are obtained by referring to tabulated values provided by a thermophysical property database [9]. The UDF utilizes this series data tables to determine the specific internal energy of a cell and all other material properties.

\section{Reproduction of Simulation Results}

To reproduce the results given in Ref. [1], the conditions of the simulation using the current implementation must match as closely as possible. Simulation results originate from a 6951 cell mesh [1]. This archived mesh is used to reproduce the following results in this research. The mesh geometry and boundary conditions are shown in Fig. 6 . The geometry is a three-dimensional 15-degree wedge section of the fuel tank. The UDF adjusts any summations to account for symmetry. All simulations are run to a flow time of 5,360 sec at a time step of $2 \mathrm{sec}$.

In cells of mixed phase fraction, the specific internal energy is calculated using Eq. (2) where $T=T_{\text {sat }}$ and is given by the reduced form Eq. (17) [1]. The specific heat of the saturated fluid is given by Eq (18).

$$
\begin{gathered}
e=c_{v} T+f \Delta e \\
c_{v}=\frac{e_{l}}{T_{s a t}}
\end{gathered}
$$

A previous time step convergence study concludes that the solution is time step independent [1]. The mesh used to obtain results is the finest mesh where data is available. Previous mesh convergence study attempts show that the solution is extremely sensitive to mesh size due to fluid density changes, which cause substantial differences in the ELF and saturation pressure. 


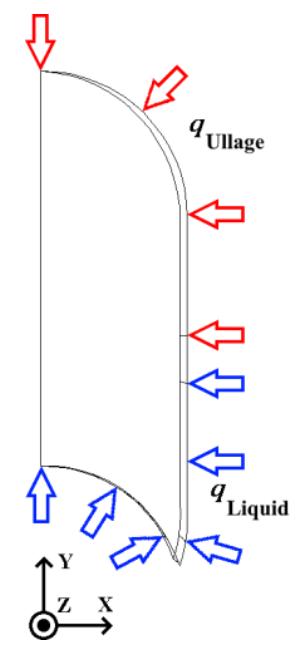

Fig. 6 AS203 fuel tank geometry and boundary conditions.

The heat flow rate within the ullage section is given by Eq. (19), and the heat flow rate within the liquid section is given by Eq. (20) [1]. These rates are divided by their respective wall areas to calculate heat flux rates for the applied boundary conditions. Certain portions of the exterior hull are set as adiabatic to avoid flash vaporization and solution divergence [1].

$$
\begin{aligned}
Q_{\mathrm{ullage}}= & -(2.855943 \mathrm{E}-14) t^{5}+(2.769605 \mathrm{E}-9) t^{4}-(1.071679 \mathrm{E}-4) t^{3}+(2.069391) t^{2} \\
& -(1.995433 \mathrm{E} 4) t+(7.693296 \mathrm{E} 7) \\
Q_{\text {liquid }}= & -(5.603975 \mathrm{E}-14) t^{5}+(5.412240 \mathrm{E}-9) t^{4}-(2.086122 \mathrm{E}-4) t^{3}+(4.013386) t^{2} \\
& -(3.855905 \mathrm{E} 4) t+(1.481028 \mathrm{E} 8)
\end{aligned}
$$

A comparison of the results obtained in Ref. [1] and the results obtained using the new implementation are shown on Fig. 7. The pressure data obtained from the experiment are also present for comparison in Fig. 7a. Pressure data points during the middle of the experiment are unavailable due to telemetry signal loss with the spacecraft. Both models predict higher pressures near the beginning of the simulation and predict lower pressures near the end. However, the current model predicts a lower pressure than that of Ref. [1].

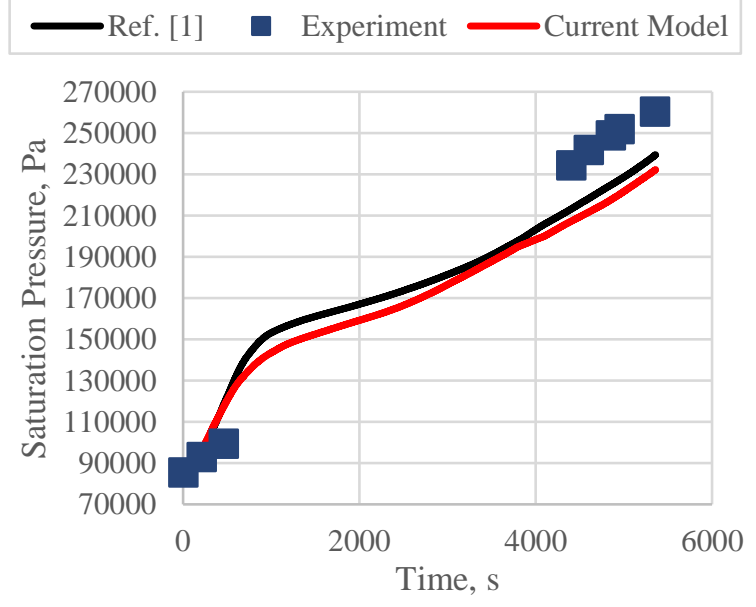

(a)

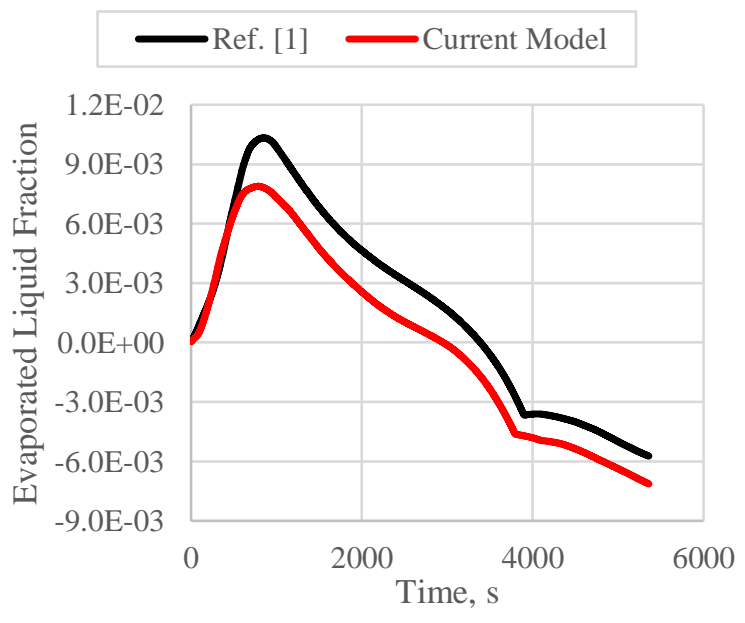

(b)

Fig. 7 Comparison of AS203 closed tank experiment simulation results: saturation pressure (a) and evaporated liquid fraction $(b)$. 
The ELF, given by Eq. (21), is a measure of the change in liquid mass from initialization. The substantial difference in the ELF and in pressure between the two simulations is likely a result of the difference in discretization of the energy equation pertaining to the thermal conductivity. In the discretization described in Ref. [1], the thermal conductivity value in the influence coefficients is a harmonic mean between the current cell and the neighboring cell. The software currently does not support this type of discretization. The difference is exacerbated where high temperature gradients are present between cells. The reason this discrepancy does not manifest in the verification cases is because the thermal conductivities are constant with respect to temperature.

$$
\mathrm{ELF}=1-\frac{m_{l}}{m_{l, \text { initial }}}
$$

Contour plots of the vapor phase fraction and temperature within the tank are shown on Fig. 8. The distribution of the phase fraction shows that superheated vapor, saturated fluid, and compressed liquid phases are all present within the tank at the same time, which is also observed in Ref. [1]. Temperature values reported by Ref. [2] shows that the ullage section should be mostly superheated vapor and should not have a large region of saturated fluid. Temperature contours captured during the simulation shows that the saturated fluid region grows directly with the increase in saturation temperature. This phenomenon is likely due to the low amount of heat transfer from the walls to the bulk fluid. More improvements are to be made to the model.

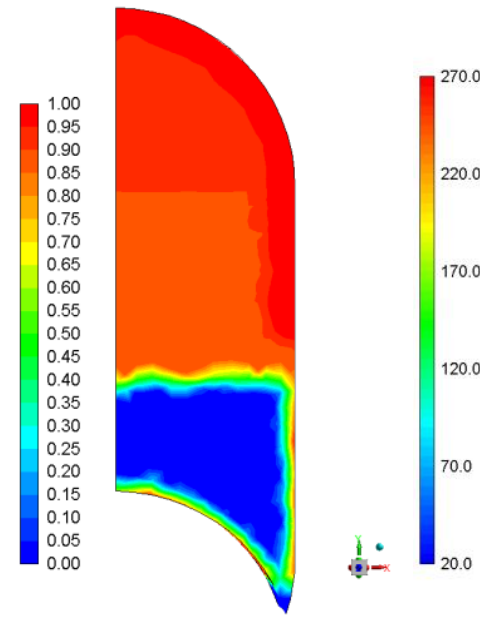

(a)

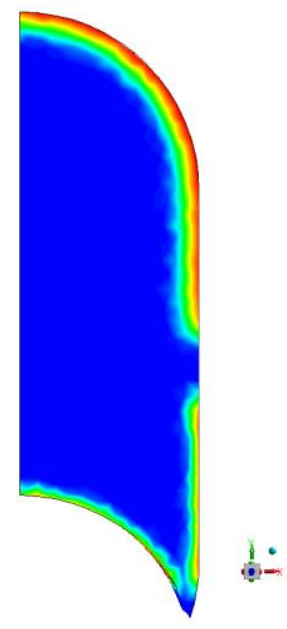

(b)

Fig. 8 Contour plots from AS203 simulation: vapor phase fraction (a) and temperature, $K$ (b).

\section{Convection Model}

Two cases are investigated where fluid flow is allowed for different phases. These models use the same mesh from the conduction only model. The same time step of $2 \mathrm{sec}$ is used for both cases. The results of the study are shown on Fig. 9. Gravitational acceleration is obtained from vehicle acceleration data from the experiment and ranges from $3.08 \mathrm{E}-3 \mathrm{~m} / \mathrm{s}^{2}$ to $7.16 \mathrm{E}-4 \mathrm{~m} / \mathrm{s}^{2}$ [3]. Only the latter value is used for the sake of simplicity. Gravity is directed towards the aft end of the tank or in the negative y-direction.

For an initial model, convection is prohibited in the liquid region. This vapor only convection model acts similarly to a solidification model. The liquid motion is suppressed starting at the interface using a porosity source term in the momentum equations, given by Eq. (22) [12]. As the vapor phase fraction approaches zero, this source term acts as a sink to the momentum equation and reduces the cell velocity to zero. The pressure rise in the model seems to follow the trends of the conduction only model until halfway into the simulation. The pressure value from this model also seems to match the pressure value of the experiment very closely at the end at 261,137 Pa. The model also predicts a boil-off in the liquid, indicated with a positive ELF value, of $79.16 \mathrm{~kg}$.

$$
S=-(1 \mathrm{E} 6) \frac{(1-f)^{2}}{f^{3}+0.001} \boldsymbol{u}
$$

For a second model, convection is allowed for both phases. This full convection model is the most unstable so far. Relaxation factors for the mass and momentum equations in the software are decreased such that a stable solution 
could be obtained. The full convection model predicts a higher fraction of evaporated liquid than the vapor only convection model.

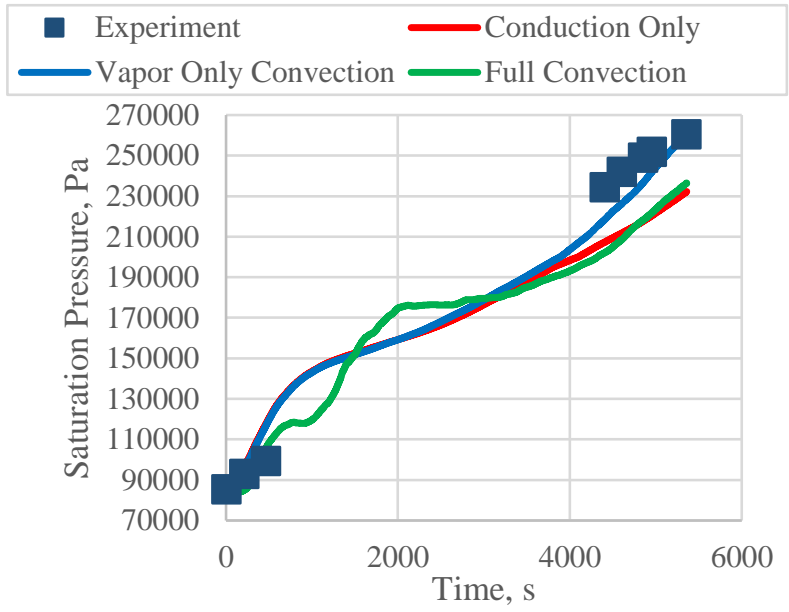

(a)

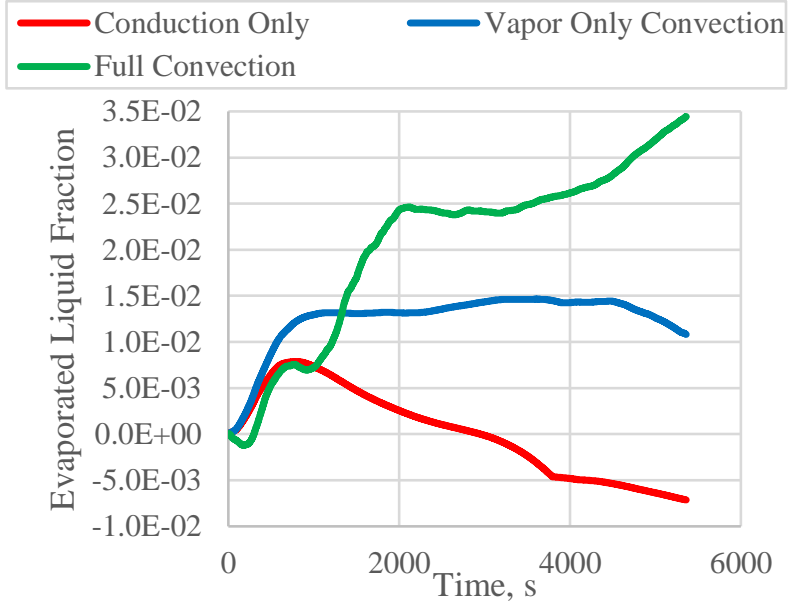

(b)

Fig. 9 Results from initial convection model simulations: saturation pressure (a) and evaporated liquid fraction (b).

\section{Conclusion}

The research presented is currently a work in progress. The code verification section shows the new implementation of the energy of fluid method produces the same results as Ref. [1] and agrees with analytical equations. The objective to simulate and reproduce the results from the simulation of the AS203 closed tank experiment is satisfied with discrepancy. This discrepancy is explained by a difference in influence coefficients that cannot be implemented into the software.

The next step is to improve upon the convection portion of the model. Future work will also investigate a convection model that suppresses the fluid motion of cells with mixed phase fractions or cells near the interface. This model aims to prevent fluid flow through the liquid-vapor interface, which is a phenomenon present in the full convection model.

Reproduction of previous results requires the use of an archived mesh. This mesh is not required for subsequent simulations. Considering the geometry of the problem, an axisymmetric geometry is to be used for future simulations. The energy equation is a scalar equation and will likely not be affected by the change in coordinate system. A twodimensional mesh allows any grid refinements to yield a smaller increase in computational cells compared to a threedimensional mesh, resulting in more efficient computational times. Time step and mesh convergence studies are conducted for a two-dimensional axisymmetric geometry of the AS203 fuel tank simulation. The studies conclude that the solution is extremely sensitive to mesh size and resulted in a $10 \mathrm{~mm}$ cell size mesh.

A proposed improvement to be implemented is how energy is transported in the unsteady and convection terms in the scalar transport equation. As previously discussed, Eq. (2) is formed based on an assumption which may not be valid near the vapor dome for hydrogen. Since the internal energy is referred from tabulated values, the proposition is made such that energy scalar transport equation uses these values directly from the data tables. The energy equation to be specified in the code is Eq. (1). Changes in internal energy due to latent heat are implicit in the tabulated values. Therefore, the unsteady and convective latent heat terms are likely unnecessary. Code verification and simulation of the AS203 tank will be reconducted using this form.

\section{Acknowledgments}

The research presented has been supported by the University of Memphis Department of Mechanical Engineering and the Tennessee Space Grant Consortium. 


\section{References}

[1] Winter, A., "Simulating Self Pressurization in Propellant Tanks Using an Energy of Fluid Approach," Dissertation, Department of Mechanical Engineering, The University of Memphis, Memphis, TN, 2014.

[2] Ward, W. D., Toole, L. E., Ponder, C. A., Meadows, M. E., Simmons, C. W. Lytle, J. H., McDonald, J. M. and Kavanaugh, B. M., "Evaluation of AS-203 Low Gravity Orbital Experiment," NASA CR 94045, Chrysler Corp Report BB-3.4.3-S-101, 1967.

[3] Bradshaw, R. D., "Evaluation and Application of Data from Low-Gravity Orbital Experiment," NASA CR $109847,1970$.

[4] Anghaie, S., and Ding, Z., "Thermal-hydraulic Analysis of Bulk Evaporation and Condensation in a Multiphase Nuclear Fuel Cell," Nuclear Technology, Vol. 120, 1997, pp. 57-70.

[5] Anghaie, S., and Ding, Z., "Modeling of Bulk Evaporation and Condensation," NASA CR-198392, 1996.

[6] Ding, Z., and Anghaie, S., "Numerical Modelling of Conduction-driven Bulk Evaporation and Condensation Processes with Constant Volume," International Journal for Numerical Methods in Engineering, Vol. 39, 1996, pp. 219-233.

[7] Myers, G. E., Analytical Methods in Conduction Heat Transfer, $2^{\text {nd }}$ Ed., AMCHT Publications, Madison, WI, 1998, p. 135.

[8] Carslaw, H. S., and Jaeger, J. C., Conduction of Heat in Solids, $2^{\text {nd }}$ Ed., Oxford Press, New York, 1959, pp. 112, $286-287$.

[9] Thermophysical Properties of Parahydrogen, National Institute of Standards and Technology Chemistry WebBook, https://webbook.nist.gov/cgi/cbook.cgi?Name=parahydrogen \&Units=SI

[10] ANSYS FLUENT 19.1, 2018.

[11] ANSYS FLUENT 19.1 User's Guide, 2021.

[12] ANSYS FLUENT 19.1 Theory Guide, 2021.

[13] ANSYS FLUENT 19.1 Customization Manual, 2021. 